\title{
Erratum to: Optical properties of uniformly sized silicon nanocrystals within a single silicon oxide layer
}

\author{
A. En Naciri • P. Miska • A.-S. Keita • \\ Y. Battie $\cdot$ H. Rinnert $\cdot$ M. Vergnat
}

Published online: 7 May 2014

(C) Springer Science+Business Media Dordrecht 2014

\section{Erratum to: J Nanopart Res (2013) 15:1538 DOI 10.1007/s11051-013-1538-0}

The authors regret that during the publication of the above paper an error occurred about the effectivemedium theory used to extract the optical responses of Si-NC (Section 3, Analysis of dielectric properties, paragraph 1). This corrected paragraph is reproduced below:

The composite silicon oxide layer, containing the $\mathrm{Si}-\mathrm{NC}$, is modeled by the Bruggeman effectivemedium approximation (BEMA) theory and not
Maxwell-Garnett theory as mentioned in the original publication. BEMA law is formulated by this equation:

$f_{\mathrm{SiO}_{2}} \frac{\varepsilon_{\mathrm{SiO}_{2}}-\varepsilon_{\mathrm{eff}}}{\varepsilon_{\mathrm{SiO}_{2}}+2 \varepsilon_{\mathrm{eff}}}+f_{\mathrm{Si}-\mathrm{NC}} \frac{\varepsilon_{\mathrm{Si}-\mathrm{NC}}-\varepsilon_{\mathrm{eff}}}{\varepsilon_{\mathrm{Si}-\mathrm{NC}}+2 \varepsilon_{\mathrm{eff}}}=0$

where $f_{\mathrm{Si}-\mathrm{NC}}$ is the volume fraction of $\mathrm{Si}-\mathrm{NC}$, and $\varepsilon_{\mathrm{SiO}_{2}}$, $\varepsilon_{\mathrm{Si}-\mathrm{NC}}$ and $\varepsilon_{\text {eff }}$ are, respectively, the dielectric functions of silicon oxide, $\mathrm{Si}-\mathrm{NC}$ and the effective medium (mixture between $\mathrm{Si}-\mathrm{NC}$ and $\mathrm{SiO}_{2}$ ).

The authors confirm that the changes mentioned in this erratum do not affect any part of the paper.

The online version of the original article can be found under doi:10.1007/s11051-013-1538-0.

A. En Naciri $(\bowtie) \cdot$ Y. Battie

LCP-A2MC, Institut Jean Barriol, Université de Lorraine,

1 Bd Arago, 57070 Metz, France

e-mail: aotmane.en-naciri@univ-lorraine.fr

P. Miska $\cdot$ H. Rinnert - M. Vergnat

Institut Jean Lamour CNRS UMR 7198,

Université de Lorraine, Bd des Aiguillettes,

B.P. 239, 54506 Vandoeuvre-lès-Nancy, France

\section{A.-S. Keita}

Max Planck Institute for Intelligent Systems,

Heisenbergstrasse 3, 70569 Stuttgart, Germany 